\title{
Inovação na tecnologia construtiva de revesti- mento com a aplicação da argamassa projetada: caracterização e indicadores
}

\author{
LORDSLEEM JR, A. C. \\ Escola Politécnica de Pernambuco \\ Universidade de Pernambuco \\ 50.720-001 - Recife, Brasil \\ acasado@poli.br
}

\author{
MORAIS, G. A. T. \\ Escola Politécnica de Pernambuco \\ Universidade de Pernambuco \\ 50.720-001 - Recife, Brasil \\ gabriela_morais@outlook.com.br
}

Resumo A mecanização da aplicação da argamassa é uma solução adequada para alcançar elevado desempenho na execução dos revestimentos. Este artigo apresenta pesquisa de estudos de caso realizada em 05 edifícios de 04 canteiros de obras, nas cidades de Recife e Jaboatão dos Guararapes, no Brasil, através da qual foi possível avaliar a perda e o consumo de argamassa, e a produtividade da mão de obra relativa à execução do revestimento interno e externo de argamassa projetada. Diante dos resultados, contatou-se variação de perda de argamassa entre $27,93 \%$ e 150,02\%, do consumo de argamassa entre $7,51 \mathrm{l} / \mathrm{m}^{2}$ e $52,91 \mathrm{l} / \mathrm{m}^{2}$ e da produtividade da mão de obra de $0,18 \mathrm{Hh} / \mathrm{m}^{2}$ a 2,06Hh/m $\mathrm{m}^{2}$, para pedreiros, e 0,48Hh/m a 0,98Hh/m $\mathrm{m}^{2}$, para serventes. Como contribuição nessa área de estudo, a metodologia adotada e os valores obtidos servirão de referência para o subsetor de edificações local e parâmetro comparativo para iniciativas semelhantes.

\footnotetext{
Abstract Mechanization the mortar is an appropriate solution to achieve high performance in implementing jackets buildings. This paper shows a research over distinct studies lead in two Brazil's cities: Recife and Jaboatão dos Guararapes, through 05 buildings of 04 sites where was possible to evaluate mortar consumption and losses, besides the labor work productivity relative to the execution of internal and external jet mortar. The results of the case study showed mortar loss variation between $27.93 \%$ and $150.02 \%$ and mortar consumption variation between $7,51 \mathrm{l} / \mathrm{m} 2$ and $52,91 \mathrm{l} / \mathrm{m} 2$. The results of labor productivity were $0.18 \mathrm{Mh} / \mathrm{m}^{2}$ and 2,06 Mh/m $\mathrm{m}^{2}$ for mason and were $0.48 \mathrm{Mh} / \mathrm{m}^{2}$ and $0.98 \mathrm{Mh} / \mathrm{m}^{2}$ for assistant mason. As a contribution in this study area, the standard methodology used and the values obtained shall serve as the initial reference for the Brazilian building construction and local comparative to other initiatives with similar characteristics.
} 


\section{Introdução}

Um dos principais desafios experimentados pela construção civil brasileira nesses últimos anos consiste em alinhar a uma maior demanda por obras à necessidade de racionalização dos serviços. A adequação da utilização dos materiais de construção, visando obter uma maior eficiência, diminui o prazo e os custos de execução dos serviços e com isso reduz os desperdícios (PINHO, 2013) ${ }^{[7]}$.

Os revestimentos de argamassa exercem influência no desempenho e durabilidade da vedação vertical, além de representar parcela expressiva dos custos de uma obra, conforme o seu padrão de acabamento. (ZANELATTO, 2012) $)^{[11]}$.

No Brasil, o sistema de revestimento com uso de argamassa é largamente utilizado. No entanto, problemas como desperdício de material, variabilidade, baixa produtividade e qualidade final do produto (PARAVISI, 2008) ${ }^{[8]}$, escassez de pessoal e redução dos prazos das obras vêm impulsionando a busca por alternativas tecnológicas que consigam superar as limitações impostas pelo processo construtivo tradicional.

Diante dessa situação, a mecanização da aplicação da argamassa, procedimento comum em países mais desenvolvidos, é citada como a solução mais adequada para alcançar um elevado desempenho na execução do revestimento interno e externo das edificações, garantindo custos e prazos controlados, além de garantia da qualidade final.

A argamassa projetada é um sistema que consiste na aplicação de argamassa através de projetores. Dois tipos de projetores são amplamente utilizados: por bombas e por spray de ar comprimido com recipiente acoplado, mais conhecido como canequinha. A projeção por bomba permite um fluxo continuo de projeção, o que permite maior qualidade e produtividade. A projeção por canequinha é um método mais simples e de menor custo, além disso, apresenta riscos menores de entupimento e dispensa o uso de argamassas especiais. Porém, como desvantagem apresenta ciclo intermitente de fluxo de projeção, de encher e esvaziar.

A mecanização da aplicação dos revestimentos de argamassa contribui para a racionalização do processo, aumentando a produtividade da mão de obra e diminuindo as perdas de material, e permite que se obtenha energia de aplicação da argamassa constante, o que diminui a variabilidade do material e possibilita potencialização da aderência da argamassa ao substrato em que é aplicada. Tendo em vista que a técnica tradicional de execução dos revestimentos de argamassa é dependente da habilidade da mão de obra. Segundo Gonçalves e Bauer $(2005)^{[6]}$, o fator humano é uma das causas da alta variabilidade no processo convencional de produção. Gonçalves (2004) ${ }^{[5]}$ destacou que ao aplicar manualmente argamassa para revestimento, o operário não consegue lançar argamassa nas partes inferiores e superiores da superfície de aplicação com a mesma força que na altura de seu tórax.

No entanto, ainda que diante de tantos aspectos positivos, poucos são os parâmetros disponíveis que atestam os ganhos em eficiência da projeção mecanizada da argamassa e subsidiem o orçamento, o projeto e a execução do revestimento das edificações. Isso se deve, sobretudo pelo fato da projeção mecanizada da argamassa ter uso ainda incipiente no Brasil, sendo uma inovação tecnológica recente na região Nordeste com reduzido número de empresas usuárias.

Diante desse contexto, a Comunidade da Construção de Recife tem se mobilizado almejando obter melhor desempenho dos métodos construtivos à base de cimento. A Comunidade da Construção é um movimento nacional que busca integrar a cadeia produtiva e aumentar o desempenho dos sistemas construtivos à base de cimento. Lançada pela Associação Brasileira de Cimento Portland (ABCP) em 2002, a Comunidade da Construção conta com a parceria dos Sinduscons nas cidades onde atua e a participação de diversas entidades nacionais e locais.

Sob a liderança da ABCP, a Comunidade da Construção tem desenvolvido programas que incentivam a racionalização dos processos construtivos nos seus ciclos de atividades. Os ciclos contemplam atividades de aprimoramento técnico e gerencial ligadas ao tema adotado e têm duração entre 12 e 24 meses.

O $6^{\circ}$ ciclo da Comunidade da Construção de Recife contemplou temas como a mecanização de revestimentos com enfoque principal nos revestimentos de argamassa aplicados com bombas de projeção.

Inserido neste contexto, a presente pesquisa foi desenvolvida junto à Comunidade da Construção da $\mathrm{ABCP}$, como parte das ações do $6^{\circ}$ ciclo de atividades (2013/2014). Diante disto, implantou-se o Programa de Indicadores de Desempenho para Tecnologias à Base de Cimento (PROGRIDE), no qual as empresas construtoras envolvidas puderam contribuir com seus resultados e informações.

O PROGRIDE foi desenvolvido no âmbito nacional da Comunidade da Construção, sob a liderança da $\mathrm{ABCP}$ e tem como objetivos principais a criação e implantação de metodologias de levantamento de indicadores como meio 
para aumento da qualidade e da produtividade das construtoras participantes e com isto a disseminação das boas práticas construtivas.

O monitoramento das perdas de materiais e produtividade da mão de obra auxiliam a identificação dos pontos críticos de processos construtivos, sendo uma importante ferramenta para otimizar a eficiência na utilização de materiais e aumentar a produtividade da mão de obra, o que leva a uma redução dos custos de produção.

\section{Objetivo}

Este artigo tem como objetivo apresentar a pesquisa de estudos de caso em 04 canteiros de obras de edificação vertical, na qual foi possível monitorar os indicadores de consumo e perda de argamassa industrializada e da produtividade da mão de obra na execução do revestimento interno e externo de argamassa por projeção mecânica.

\section{Metodologia}

A metodologia adotada para a consecução deste trabalho contemplou as etapas:

1. revisão bibliográfica: identificando a literatura sobre mecanização da projeção de argamassa; coleta de perdas, consumo e produtividade e a existência de valores de referência;

2. definição de metodologia de coleta de dados e dos elementos operacionais;

3. pesquisa de estudos de caso: acompanhamento sistemático dos indicadores gerados em 04 canteiros de obras de edifícios multipisos;

4. compilação e análise dos resultados: análise comparativa com os valores de referência.

No item seguinte serão caracterizadas as fórmulas de cálculo e a metodologia de coleta dos indicadores utilizados para o desenvolvimento desta pesquisa.

\section{Indicadores de perdas e produtivi- dade}

Os indicadores implementados na pesquisa e suas respectivas fórmulas de cálculo e metodologia de coleta dos indicadores serão apresentados a seguir:

1) Perda da argamassa industrializada PAI (\%) $=[($ Creal - Cteórico $) \times 100] \div$ Cteórico Metodologia de Coleta:
Inicialmente, de posse dos dados fornecidos pelo fabricante, foi coletado o valor estabelecido pelo mesmo para o consumo de referência teórico. No primeiro dia da semana de coleta foi feito o levantamento do número de sacos de argamassa estocados no canteiro [EST (VI)] e no último dia, o total de sacos que permaneceram no estoque [EST (VF)], bem como o quantitativo de sacos recebidos ou destinados a outros serviços durante a semana em questão [FORN]. Com base nos valores obtidos, foram calculados os consumos real e teórico de argamassa. No primeiro dia de coleta foi verificado também o serviço de revestimento executado anteriormente ao início do levantamento de dados para que no último dia da semana de coleta fosse verificada área executada apenas no período.

2) Consumo unitário real de argamassa industrializada CUMr $\left(\mathrm{kg} / \mathrm{m}^{2}\right)=\operatorname{ARG}(\mathrm{kg}) \div \mathrm{QS}\left(\mathrm{m}^{2}\right)$ Metodologia de Coleta:

Para a determinação do consumo unitário real de argamassa foi necessário contabilizar o total de sacos de argamassa gastos durante uma semana e fazer o levantamento da área executada no mesmo período.

3) Razão Unitária de Produção Semanal RUPsem $\left(\mathrm{Hh} / \mathrm{m}^{2}\right)=\mathrm{Hh}(\mathrm{s}) \div \mathrm{QS}(\mathrm{s})$ Metodologia de Coleta:

Para determinar a produtividade foi necessário fazer o levantamento da quantidade de serviço executada durante o período semanal, bem como das horas trabalhadas no serviço de emboço pelos serventes e pedreiros.

4) Razão Unitária de Produção Cumulativa RUPcum $\left(H h / \mathbf{m}^{2}\right)=\Sigma H h \div \Sigma Q S$ Metodologia de Coleta:

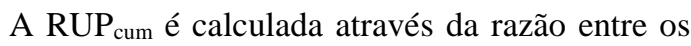
somatórios dos homens-horas demandados e das quantidades de serviços executadas semanalmente acumulados durante todo o período de estudo. Este dado é computado semanalmente.

5) Razão Unitária de Produção Potencial RUP pot $\left(\mathrm{Hh} / \mathbf{m}^{2}\right)=$ Mediana das RUPsem menores que a RUP cum final Metodologia de Coleta:

Segundo Dantas (2011) ${ }^{[4]}$, a RUP potencial não está associada a cada dia de trabalho, a mesma in- 
dica uma produtividade potencialmente alcançável desde que, mantido um determinado conteúdo de trabalho, não se tenha problemas quanto à gestão do mesmo. Obtêm-se matematicamente calculando a mediana dos valores de RUP diária inferiores ao valor da RUP cumulativa para o final do período de estudo.

Para melhor compreensão das fórmulas de cálculo apresentadas, cabe explicar o significado dos seguintes símbolos:

$\mathbf{C}_{\text {real }}=$ Consumo real $[\mathrm{Kg}]$

$\mathrm{C}_{\text {real }}=\quad \mathrm{ARG}=\{[\mathrm{EST}(\mathrm{VI})]+$ FORN $-[\operatorname{EST}(\mathrm{VF})]\} \mathrm{x}$ $\mathbf{P}$

ARG = Quantidade total de argamassa consumida no período [kg]

EST $($ VF $)=$ Quantidade de sacos de argamassa no estoque final

EST $($ VI $)=$ Quantidade de sacos de argamassa no estoque inicial

FORN = Quantidade de sacos de argamassa recebidos entre VI e VF

$\mathbf{P}=$ Peso do saco de argamassa $[\mathrm{kg}]$

$\mathbf{C}_{\text {teorico }}=$ Consumo teórico $[\mathrm{kg}]$

$C_{\text {teorico }}=C_{\text {ref }} \times e_{c} \times$ QS

$\mathbf{C}_{\text {ref }}=$ Consumo de referência do fabricante $\left[\mathrm{kg} / \mathrm{m}^{2 *} \mathrm{~cm}\right]$ para $1 \mathrm{~cm}$ de espessura da camada de revestimento

$\mathbf{e}_{\mathbf{c}}=$ Espessura da camada de revestimento $[\mathrm{cm}]$

QS = Quantidade de serviço $\left[\mathrm{m}^{2}\right]$

$\mathbf{Q S}=\Sigma \mathbf{A p}-\mathbf{\Sigma A a}$

$\mathbf{A}_{\mathbf{p}}=$ Área de parede executada (largura x altura) $\left[\mathrm{m}^{2}\right]$

$\mathbf{A}_{\mathbf{a}}=$ Área de abertura (vãos) $\left[\mathrm{m}^{2}\right]$

$\mathbf{H h}(\mathbf{s})=$ Total de Homem horas trabalhadas semanal [Hh]

QS(s) = Quantidade de serviço semanal $\left[\mathrm{m}^{2}\right]$

$\boldsymbol{\Sigma H h}=$ Somatório de Homem hora durante o período de coleta [Hh]
$\boldsymbol{\Sigma Q S}=$ Somatório de Quantidade de Serviço durante o período de coleta $\left[\mathrm{m}^{2}\right]$

Nesta pesquisa foram aplicados quatro formulários pertencentes ao Manual de indicadores de Produtividade e Perdas de Processos à Base de Cimento, aprimorados por Pinho (2013) ${ }^{[7]}$ em parceria com a Comunidade da Construção. O manual visa fornecer informações sobre a metodologia de coleta de dados, caracterizar a empresa, o empreendimento e os serviços em estudo, bem como facilitar o processamento e análise dos dados por parte dos responsáveis pelas coletas (PINHO, 2013 $)^{[7]}$. Na metodologia deste trabalho, foram aplicados os cadernos de Caracterização da empresa, Caracterização do empreendimento, Perdas de argamassa industrializada - emboço externo e Produtividade de revestimento externo - emboço.

\section{Apresentação e análise dos resulta- dos}

A caracterização das obras e dos serviços analisados precede a apresentação e análise dos resultados por ser fundamental para compreensão dos mesmos.

As obras foram codificadas por dois caracteres, sendo o primeiro uma letra e o segundo um algarismo. Foram utilizadas as letras B e C, para o caso de aplicação com bomba de projeção e canequinha, respectivamente. Os algarismos, de 1 a 5 , identificavam cada uma das obras.

\subsection{Caracterização das obras}

Os levantamentos de dados em campo foram realizados em 04 canteiros de obras, alguns dos quais com mais de um edifício em construção, pertencentes a 04 diferentes construtoras. As características das obras onde foram realizados os estudos de caso estão especificadas na Tabela 1.

\subsection{Caracterização do serviço}

Neste item, será caracterizado apenas o serviço de emboço externo em argamassa. O procedimento para a execução do serviço é descrito na sequência:

1) Para o início da execução do revestimento foi realizada a limpeza da base e a conferência do prumo da superfície.

O procedimento de revestimento com argamassa é constituído usualmente da execução de três camadas: chapisco, emboço e acabamento;

2) A primeira camada executada foi o chapisco, o qual tem a função de regular a absorção de água pela base e criar um substrato de aderência para a fixação da argamassa; 
3) A segunda camada executada foi o emboço, o qual é formado por um conjunto de operações, são elas:

- $\quad 1^{\mathrm{a}}$ operação - colocação das taliscas: as taliscas são pequenas peças de material cerâmico colocados sobe a superfície a ser revestida e que servem de referência para o acabamento. $\mathrm{O}$ taliscamento foi executado manualmente em todas as obras.

- $\quad 2^{\mathrm{a}}$ operação - execução das mestras: após o taliscamento, o espaço entre as taliscas é preenchido com a mesma argamassa do emboço. Após o endurecimento da argamassa, com o uso de uma régua de alumínio, aprumam-se as mestras, as quais servirão de guia para a execução do revestimento. As mestras foram executadas manualmente em todas as obras.

- $\quad 3^{a}$ operação - aplicação do revestimento de argamassa: após um tempo mínimo de 24 horas de endurecimento das mestras, executa-se o preenchimento dos vãos entre as mesmas com argamassa de revestimento.

Nas obras, a argamassa era misturada em argamassadeiras, no caso de aplicação com canequinha, ou em misturadores contínuos no caso da aplicação mecanizada através de bombas.

- $4^{\mathrm{a}}$ operação - sarrafeamento: é realizado usando uma régua de baixo para cima, retirando o excesso de material chapeado.

- $\quad 5^{\mathrm{a}}$ operação - desempeno: dependendo do acabamento desejado pode-se executar o desempeno da superfície com desempenadeira de mão adequada para cada caso (madeira, aço ou feltro). No caso desta pesquisa, todas as obras utilizaram desempenadeira de aço.

A última etapa da execução do serviço é o acabamento, o qual é realizado após a cura do revestimento. No caso das obras nas quais foram feitos estudos de caso, o acabamento era composto por pintura, cerâmica ou placas de granito. O tempo de espera entre a cura do revestimento e a execução do acabamento, variou de $72 \mathrm{hrs}$, para o caso de revestimento cerâmico, a 15 dias para placas de granito.

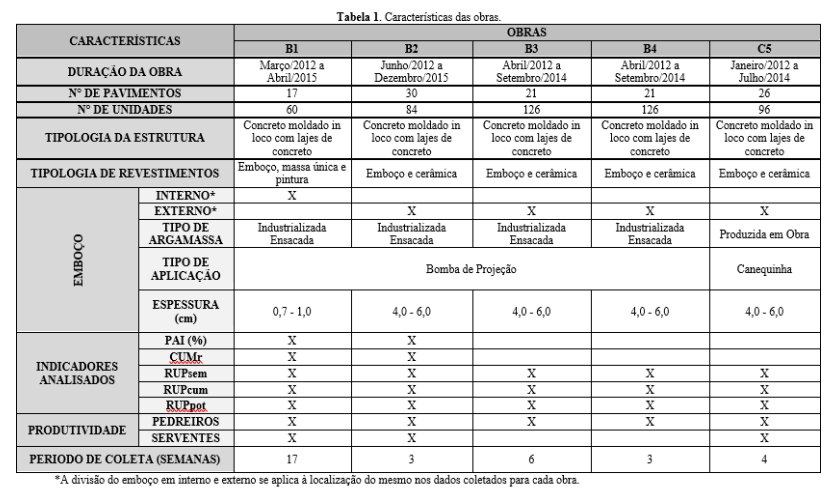

\subsection{Apresentação de resultados}

Para o melhor entendimento deste item destaca-se que o valor do consumo unitário de argamassa de referência foi pesquisado entre os fabricantes de argamassa, tendo valor de $17 \mathrm{Kg} / \mathrm{m}^{2}$ a cada $1 \mathrm{~cm}$ de espessura da camada de revestimento em todas as obras.

Para expressar os valores de consumo unitário real de argamassa em $1 / \mathrm{m}^{2}$ considerou-se cada $1 \mathrm{Kg} / \mathrm{m}^{2}$ de revestimento equivalente a $0,491 / \mathrm{m}^{2}$ de argamassa.

Os resultados mínimos, máximos, médios e medianos de todos os indicadores coletados estão dispostos na Tabela 2 .

Tabela 2. Indicadores coletados.

\begin{tabular}{|c|c|c|c|c|c|c|}
\hline \multirow{2}{*}{\multicolumn{2}{|c|}{$\frac{\text { EMBOÇO }}{\text { OBRAS }}$}} & \multirow{2}{*}{$\frac{\text { Interno }}{\text { B1 }}$} & \multicolumn{4}{|c|}{ Externo } \\
\hline & & & B2 & B3 & B4 & C5 \\
\hline \multicolumn{2}{|c|}{ Aplicação } & \multicolumn{4}{|c|}{ Bomba de Projeção } & Canequinha \\
\hline \multirow{4}{*}{$\begin{array}{l}\text { PAI } \\
(\%)\end{array}$} & Mínima & 27,93 & 28,85 & $\mathrm{NC}$ & $\mathrm{NC}$ & $\mathrm{NC}$ \\
\hline & Média & 81,23 & 41,82 & $\mathrm{NC}$ & $\mathrm{NC}$ & $\mathrm{NC}$ \\
\hline & Máxima & 150,02 & 58,74 & $\mathrm{NC}$ & $\mathrm{NC}$ & $\mathrm{NC}$ \\
\hline & Mediana & 71,32 & 37,87 & $\mathrm{NC}$ & $\mathrm{NC}$ & $\mathrm{NC}$ \\
\hline \multirow{4}{*}{$\begin{array}{c}\text { CUMr } \\
\left(1 / \mathbf{m}^{2}\right)\end{array}$} & Mínimo & 7,51 & 42,95 & $\mathrm{NC}$ & $\mathrm{NC}$ & $\mathrm{NC}$ \\
\hline & Médio & 11,63 & 47,27 & $\mathrm{NC}$ & $\mathrm{NC}$ & $\mathrm{NC}$ \\
\hline & Máximo & 16,67 & 52,91 & $\mathrm{NC}$ & $\mathrm{NC}$ & $\mathrm{NC}$ \\
\hline & Mediano & 11,42 & 45,96 & $\mathrm{NC}$ & $\mathrm{NC}$ & $\mathrm{NC}$ \\
\hline \multirow{4}{*}{$\begin{array}{c}\text { RUPsem } \\
\left(\mathbf{H h} / \mathbf{m}^{2}\right)\end{array}$} & Mínima & 0,21 & 0,7 & 0,18 & 0,23 & 0,43 \\
\hline & Média & 0,86 & 1,44 & 0,26 & 0,42 & 0,71 \\
\hline & Máxima & 2,06 & 1,81 & 0,34 & 0,75 & 1,17 \\
\hline & Mediana & 0,69 & 1,8 & 0,27 & 0,37 & 0,61 \\
\hline RUPcum & Mínima & 0,41 & 0,7 & 0,18 & 0,23 & 0,47 \\
\hline
\end{tabular}




\begin{tabular}{|c|c|c|c|c|c|c|}
\hline $\left.\mathbf{( H h} / \mathbf{m}^{2}\right)$ & Média & 0,51 & 1,24 & 0,22 & 0,42 & 0,55 \\
\cline { 2 - 7 } & Máxima & 0,58 & 1,56 & 0,25 & 0,49 & 0,61 \\
\cline { 2 - 7 } & Mediana & 0,51 & 1,45 & 0,24 & 0,44 & 0,56 \\
\hline $\begin{array}{c}\mathbf{R U P} \\
\left.\mathbf{( H h}_{\text {pot }} \mathbf{m}^{2}\right)\end{array}$ & 0,37 & 0,7 & 0,18 & 0,28 & 0,48 \\
\hline
\end{tabular}

* NC - Dado não coletado

*RUP's de pedreiros

\subsection{Análise dos resultados}

Neste item foi feita análise dos resultados obtidos em comparação com os valores de referência de outros trabalhos que estudaram o mesmo tema. A análise dos resultados da obra B1 foi feita separadamente das demais obras por ser esta a única onde a localização do revestimento é interno.

\subsubsection{Análise dos resultados}

A perda de argamassa foi analisada nas obras B1 e B2. A obra B1 teve acompanhamento de 17 semanas, a obra B2 teve acompanhamento de 03 semanas.

\subsubsection{Analise da perda percentual na obra B1}

Os seguintes valores de referência devem ser utilizados para a comparação dos resultados:

1) AGOPYAN ET AL (1998) $)^{[1]}$ - pesquisa realizada em 12 obras distintas com perdas de argamassa industrializada no serviço de emboço interno variando entre $5 \%$ e $209 \%$;

2) BEZERRA E MERGULHÃO (1998) $)^{[2]}$ - pesquisa realizada em 03 obras distintas com perdas de argamassa no serviço de emboço interno variando entre $53,87 \%$ e $63,15 \%$.

Pôde-se constatar que a média de perda de argamassa na obra B1 está compreendida no intervalo de valores encontrados por Agopyan et al (1998) ${ }^{[1]}$ e foi superior aos valores coletados por Bezerra e Mergulhão (1998) ${ }^{[2]}$. No entanto, ainda que compreendida entre o intervelo de valores apresentados em pesquisas anteriores, o valor médio e mediano da perda é consideravelmente elevado. Fatores como a falta de revestimento no piso do local onde o emboço era executado, a existência de vigas mais largas que a alvenaria, o desaprumo e irregularidade da alvenaria, a inexistência de política de reaproveitamento da argamassa que cai no chão, o não incentivo à redução das perdas e a baixa capacitação da mão de obra contribuíram para elevar os valores das perdas.

\subsubsection{Análise da perda percentual na obra B2}

Os seguintes valores de referência devem ser utilizados para a comparação dos resultados:

1) SOIBELMAN (1993) ${ }^{[9]}$ - pesquisa realizada em 03 obras distintas com perdas de argamassa industrializada no serviço de emboço externo variando entre $10,5 \%$ e $225 \%$ e com perda média de $76,5 \%$;

2) $\operatorname{COSTA}(2005)^{[3]}$ - pesquisa realizada em 08 construtoras distintas com perdas de argamassa no serviço de emboço externo variando de $66 \%$ a $99 \%$ com média de $81 \%$.

O valor de perda média percentual encontrado na obra B2 está compreendida no intervalo de valores encontrados por Soibelman (1993) ${ }^{[9]}$ e abaixo daquele coletado por Costa $(2005)^{[3]}$; além disso, foi inferior ao valor determinados pelos referidos autores para perda média.

Fatores como o desaprumo e a pequena extensão da superfície a ser revestida, a inexistência de política de reaproveitamento da argamassa que cai, o não incentivo à redução das perdas e a baixa capacitação da mão de obra contribuíram para elevar os valores das perdas.

\subsubsection{Análise do consumo unitário real de argamassa}

O consumo unitário real de argamassa foi analisado nas obras B1 e B2, as obras tiveram acompanhamento de 17 e 03 semanas, respectivamente.

\subsubsection{Análise do consumo unitário real de argamassa na obra $B 1$}

Considerando os valores propostos pelo TCPO 14 (PINI, 2012) $)^{[10]}$, que apresenta para o serviço de emboço interno valor de consumo unitário de argamassa mínimo de $7,31 / \mathrm{m}^{2}$, mediano de $21,91 / \mathrm{m}^{2}$ e máximo de $59,31 / \mathrm{m}^{2}$, pode-se verificar que a obra $\mathrm{B} 1$ teve valor de consumo mediano inferior e consumo médio bastante próximo ao mínimo proposto pelo TCPO 14 (PINI, 2012) ${ }^{[10]}$.

\subsubsection{Análise do consumo unitário real de argamassa na obra $B 2$}

Considerando os valores propostos pelo TCPO 14 (PINI, 2012) $)^{[10]}$, que apresenta para o serviço de emboço externo valor de consumo unitário de argamassa mínimo de $201 / \mathrm{m}^{2}$, mediano de $351 / \mathrm{m}^{2}$ e máximo de $661 / \mathrm{m}^{2}$, pode- 
se verificar que a obra a obra B2 obteve valores satisfatórios, tendo seu valor médio de consumo próximo ao mínimo estabelecido pelo TCPO 14 (PINI, 2012) ${ }^{[10]}$.

\subsubsection{Análise da produtividade na obra}

A produtividade da mão de obra foi analisada nas obras $\mathrm{B} 1, \mathrm{~B} 2, \mathrm{~B} 3, \mathrm{~B} 4$ e C5, as obras tiveram acompanhamento de 17, 03, 03, 06 e 04 semanas, respectivamente.

\subsubsection{Análise do consumo unitário real de argamassa na obra $B 1$}

Os valores encontrados para as RUP's semanais da obra B1 apresentaram grande variabilidade. Considerando os valores propostos pelo TCPO 14 (PINI, 2012) ${ }^{[10]}$, que apresenta para o serviço de emboço interno valor mínimo de produtividade para os pedreiros de $0,41 \mathrm{Hh} / \mathrm{m}^{2}$, mediano de $0,57 \mathrm{Hh} / \mathrm{m}^{2}$ e máximo de $0,98 \mathrm{Hh} / \mathrm{m}^{2}$, verifica-se que apesar de apresentar valores de produtividade bastante superiores ao valor máximo estabelecido pelo TCPO 14 (PINI, 2012) ${ }^{[10]}$, as RUP's semanais dos pedreiros se mantiveram satisfatórias na maior parte do período de coleta.

Os resultados apresentados da obra B1 para RUP cumulativa dos pedreiros demonstram que houve uma melhoria na produtividade dos mesmos ao longo das primeiras semanas, no entanto ao longo do tempo houve um au-

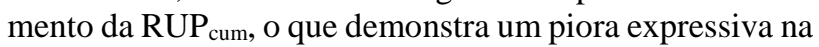
produtividade. A RUP ${ }_{\text {cum }}$ dos pedreiros está representada no Gráfico 1.

Gráfico 1. RUP $P_{\text {cum }}$ dos pedreiros na obra $\mathrm{B} 1$.

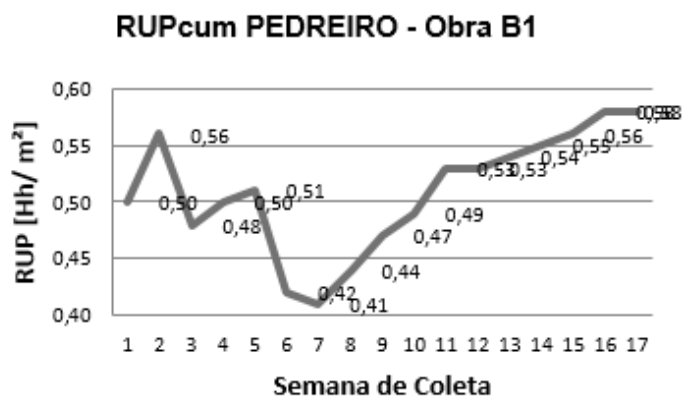

Fatores como a elevada quantidade de retrabalho, o acabamento desempenado, a execução manual dos capiaços e taliscas, problemas na produção da argamassa, a alta rotatividade, o atraso na remuneração e a baixa capacitação da mão de obra contribuíram para piora da produtividade.

\subsubsection{Análise de produtividade da mão de obra nas obras B2, B3, B4 e C5}

Considerando os valores propostos pelo TCPO 14 (PINI, 2012) ${ }^{[10]}$, que apresenta para o serviço de emboço externo valor mínimo de produtividade para os pedreiros de $0,40 \mathrm{Hh} / \mathrm{m}^{2}$, mediano de $0,79 \mathrm{Hh} / \mathrm{m}^{2}$ e máximo de $2,60 \mathrm{Hh} / \mathrm{m}^{2}$, pode-se verificar que a obra B2 teve os piores resultados; enquanto, a obra B3 obteve os valores mais satisfatórios. A obra B4 obteve resultados satisfatórios, bem como a obra $\mathrm{C} 5$, porém, sendo os valores desta última aquém dos obtidos na obra B4.

Os resultados apresentados para RUP cumulativa dos pedreiros demonstram uma tendência de piora em relação ao tempo. A obra B4 apresenta melhoria de produtividade pouco expressiva. A obra B2 teve os piores resultados em longo prazo. As obras B 3 e C5 apresentaram piora na produtividade ao longo do tempo. A RUP cum $_{\text {dos }}$ pedreiros no serviço de emboço externo nas obras B2, B3, B4, C5 e M6 está representada no Gráfico 2.

Gráfico 2. RUP $\mathrm{Pum}_{\text {cum }}$ dos pedreiros nas obras B2, B3, B4 e C5.

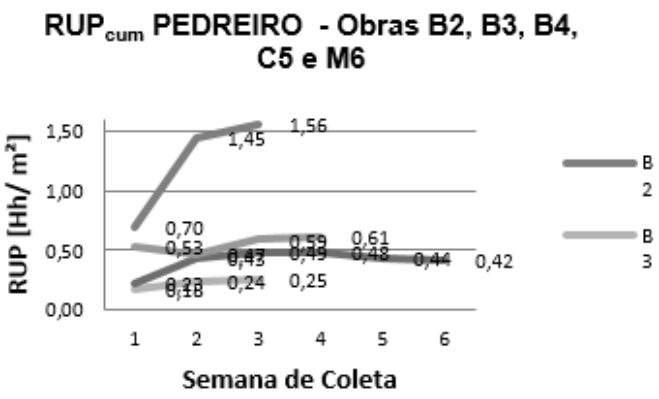

Fatores como a execução manual dos capiaços, taliscas e mestras, problemas na produção da argamassa, falta de água, quebra de equipamentos, a alta rotatividade e a baixa capacitação da mão de obra contribuíram para piora da produtividade.

\section{Conclusão}

A utilização da projeção mecânica da argamassa não provocou redução significativa nas perdas de argamassa e no prazo de execução das obras. Mesmo investindo nos equipamentos necessários para a execução dos revestimentos, as empresas não obtiveram resultados expressivos quanto à eficiência do sistema. O que se deve, sobretudo, à falta de planejamento do processo de produção. No entanto, é difícil mensurar os benefícios obtidos em função da maior qualidade do produto proporcionada pelos sistemas mecanizados de projeção. 
A metodologia proposta neste artigo constitui-se uma ferramenta auxiliar na avaliação quantitativa da tecnologia construtiva de revestimento com a aplicação da argamassa projetada, permitindo ao usuário analisar os parâmetros para utilizar a projeção de argamassa na construção civil.

A aplicação da metodologia apresentada nesta pesquisa permitiu às empresas construtoras envolvidas, visualizarem a ocorrência de perdas através dos indicadores de desempenho aplicados e observarem a necessidade de mudanças com vistas a alcançar a racionalização dos serviços.

\section{Referências}

[1] AGOPYAN, V. et al. Pesquisa: Alternativas para a redução do desperdício de materiais nos canteiros de obras. Relatório final. EPUSP/FINEP/ITQC, 1998.

[2] C. S. B. Bezerra; R.A. C. Mergulhão. Aspectos qualitativos e quantitativos das perdas de materiais empregados no revestimento com argamassa produzido nas obras. In: XVIII Encontro Nacional de Engenharia de Produção, Niterói, 1998. Anais... Niterói, 1998.

[3] COSTA, F. N. Processo de Execução de Revestimento de Fachada de Argamassa: Problemas e Oportunidades de Melhoria. 2005. 195 p. Dissertação (Mestrado) - Universidade Federal do Rio Grande do Sul. Porto Alegre, 2005.

[4] DANTAS, J.D.F. Produtividade da mão de obra - Estudo de caso: métodos e tempos na indústria da construção civil no subsetor de edificações na cidade de João Pessoa- PB. 2001. 68p. Tese de Conclusão de Curso - Universidade Federal da Paraíba. João Pessoa, 2011.

[5] GONÇALVES, S. R. de C. Variabilidade e fatores de dispersão da resistência de aderência nos revestimentos em argamassa - estudo de caso. 2004. 148p. Dissertação (Mestrado em Estruturas e Construção Civil) - Universidade de Brasília, Brasília.

[6] GONÇALVES, S. R. de C.; BAUER, E. Estudo de caso da variação de resistência de aderência à tração em uma parede. In: VI Simpósio Brasileiro de Tecnologia de Argamassas, Florianópolis, 2005. Anais... Florianópolis, 2005, p. 562-567.

[7] PINHO, S.A.C. Desenvolvimento de programa de indicadores de desempenho para tecnologias construtivas à base de cimento: perdas, consumo e produtividade. 2013. 268p. Dissertação (Mestrado) - Escola Politécnica, Universidade de Pernambuco. Recife, 2013.

[8] PARAVISI, S. Avaliação de sistemas de produção de revestimento de fachada com aplicação mecânica e manual de argamassa. 2008. 179 p. Dissertação (Mestrado) - Escola de Engenharia, Universidade Federal do Rio Grande do Sul. Porto Alegre, 2008.

[9] SOIBELMAN, L. As perdas de materiais na construção de edificações: sua incidência e seu controle. Porto Alegre, 1993. 127p. Dissertação (Mestrado em engenharia) - Universidade Federal do Rio Grande do Sul.

[10] TCPO. Tabelas de Composição de Preços para Orçamentos. 14. ed. São Paulo: Pini, 2012. ISBN 978-857266-251-2

[11] ZANELATTO, K.C. Avaliação da influência da técnica de execução no comportamento dos revestimentos de argamassa aplicados com projeção mecânica contínua. 2012. 142p. Dissertação (Mestrado) - Escola Politécnica, Universidade de São Paulo. São Paulo, 2012. 